\title{
Analysis of Over-sampling based Identification *
}

\author{
Mengyuan Fang ${ }^{\text {a }}$, Yucai Zhu ${ }^{\text {a }}$ \\ ${ }^{a}$ State Key Laboratory of Industrial Control Technology, College of Control Science and Engineering, Zhejiang University \\ Zheda Road 38, Hangzhou 310027, China
}

\begin{abstract}
In this work, firstly, an asymptotic variance expression is derived for the transfer function estimates in the over-sampling based identification scheme. Then the result is used to analyze the over-sampling scheme. The asymptotic variance expression says that the joint covariance matrix of the transfer function estimates is proportional to the "generalized" noise-to-signal ratio. It is an application of the result in Ljung (1985); and it covers both open-loop and closed-loop tests. Using the result, one can show that informativity of the closed-loop tests without external excitation can be attained by using the over-sampling scheme, but only when the output disturbance has high frequency content beyond the bandwidth of the plant. Moreover, when the output disturbance has high frequency content and when test signal is used, the result points out that the over-sampling scheme can increase model accuracy, and, it will outperform conventional method with anti-aliasing filtering. Numerical examples are used to illustrate the asymptotic variance expression and the analysis about over-sampling scheme of identification.
\end{abstract}

Key words: Asymptotic variance, over-sampling, closed-loop identification, transfer functions

\section{Introduction}

System identification plays the most important role in model-based control applications such as model predictive control (MPC) and robust control. In last decades, many identification algorithms have been developed and analyzed, see Ljung (1999), Söderström and Stoica (1989). The basic requirement for system identification, especially in large-scale control applications, is to obtain good quality models at a low cost. In most industrial applications, the cost of identification is related to the disturbance caused by the test signals and to the identification test time. Hence to reduce the cost of identification implies the reduction of the disturbance of identification test and the test time. The test signal is generally restricted to a considerably low level in many practical systems, especially for MPC identification of large and capital intensive industrial processes such as distillation columns and power plants where the identification excitation should not cause any off spec of the products, or, disturb normal operation, see Zhu (2001), and this may lead to poor model quality and control

\footnotetext{
‡ This work is supported by the National Science Foundation of China (No. 61273191) and by 973 Program of China (No. 2012CB720500). Corresponding author Yucai Zhu. Tel. +15381085960. Fax +0571-87953353.
}

Email addresses: myfang@iipc.zju.edu.cn (Mengyuan Fang), yczhu@iipc.zju.edu.cn (Yucai Zhu). performance. The situation can be improved when identification test is carried out in closed-loop under feedback control because the controller can reduce the output variations considerably. The cost of computing power and computing time can be neglected in off-line model identification.

Inspired by the over-sampling technique used in signal processing area, in Sun et al. (1997) and Sun et al. (2001) the over-sampling based identification scheme has been proposed, and it is proved in time domain that this scheme can attain informative experiments ${ }^{1}$ in closedloop identification without external excitation, which means informative data can be obtained from noise excitation only and the open-loop plant can be identified with no test signals applied. Sun et al. (2000) proposed a frequency domain closed-loop identification algorithm using the over-sampling scheme which can also attain informative closed-loop experiments without external excitation. In Wang et al. (2004) and Sun and Sano (2009), the authors make use of the cyclo-stationarity of the over-sampled signals to prove in frequency domain that the observed data in over-sampling based closed-loop identification without external excitation is informative. In Sun and Zhu (2012), an over-sampling based closedloop identification algorithm is investigated which can

\footnotetext{
1 For definition of informative experiments, see Definition 8.1 in Ljung (1999).
} 
reduce the influence of sensitivity to the noise model estimation and initial values in the numerical optimization.

We would like to mention that the over-sampling technique is practically costless. In industrial MPC (model predictive control) systems, the controller sampling frequency is much lower than the sampling frequency of the lower level DCS (distributed control system). For example, the sampling time of a typical refinery DCS system is 1 second or shorter, while the sampling time of a typical MPC control system is 60 seconds. This means that in MPC identification, the sampling frequency can be made 60 times as high if necessary. The situations in other industries are similar. Because no test signal is used, the over-sampling scheme is the most economical test approach, namely, the cost is practically zero.

However, the over-sampling based identification scheme has its shortcomings and the model quality is not guaranteed: the quality of identified plant model is strongly dependent on controller parameters, the positions of plant poles and the property of unmeasured disturbance. In Sun and co-workers' works, the external excitation is set to zero in the identification tests. In industrial applications, external test signals are permitted although they should be kept small in amplitudes.

Here, in order to achieve high model quality and low disturbing test, we propose the use of external test signal in the over-sampling scheme in identification tests. We start by analyzing the asymptotic properties of the transfer function estimates in over-sampling based identification where external excitation is used, which includes the situation without excitation as a special case. Variance expression asymptotic both in the number of observed data and in the model order is derived and has an explicit form. This is an application of Ljung (1985) to the over-sampling based identification. The results cover open-loop tests which has not been considered in the previous study of Sun and co-workers. Using the result, we can give the exact condition for informative closed-loop test without excitation for the over-sampling scheme which is previously unknown. Moreover, we can show that, when test signal is used, under the same condition, the over-sampling scheme can increase model accuracy in both open-loop and closed-loop identification.

The outline of this paper is as follows. In Section 2, the over-sampling technique is briefly introduced; the asymptotic variance expression in over-sampling based identification is derived in Section 3; Section 4 gives the analysis of the over-sampling based identification using the asymptotic variance expression; numerical illustrations are given in Section 5 and Section 6 contains the conclusion and perspective.

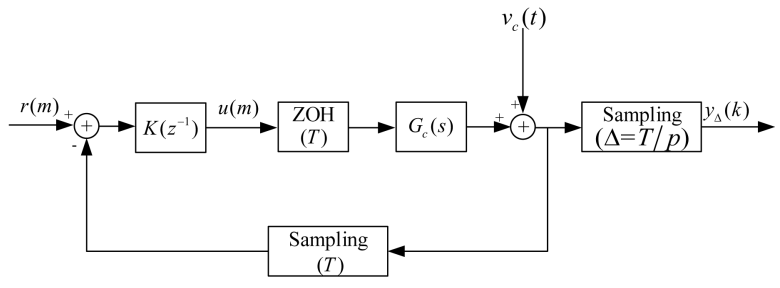

Fig. 1. Over-sampling technique in closed-loop identification

\section{Over-sampling technique}

Fig. 1 displays how the over-sampling technique is used in closed-loop identification. Same as in conventional closed-loop identification, the closed-loop system contains a linear continuous-time plant $G_{c}(s)$ and a digital controller $K\left(z^{-1}\right)$ of which the control period is $T$, and $z^{-1}$ is a backwards shift operator corresponding to $T$. $K\left(z^{-1}\right)$ is followed by a zero-order holder to generate piecewise control input. The difference is that the output is sampled at a period of $\Delta=T / p$ to generate the signal $y_{\Delta}(k)$ used for identification while in conventional identification the sampling time for output signal equals $T$. Here $p$ is a positive integer indicating the over-sampling rate. Notice that the input is also oversampled. But due to the zero-order holding, the input signal $u_{\Delta}(k)$ for identification is actually generated as

$u_{\Delta}(k)=u(m), k=m p, m p+1, \cdots(m+1) p-1$.

Due to the zero-order holding, the over-sampled input and output, i.e. $u_{\Delta}(k)$ and $y_{\Delta}(k)$, are cyclo-stationary signals for which the cyclo-stationary correlation function is defined as

$C_{x_{1}, x_{2}}\left(\alpha_{l}, \tau\right)=\frac{1}{p} \sum_{k=m p}^{m p+p-1} e^{i \alpha_{l} k} E\left\{x_{1}(k+\tau) x_{2}(k)\right\}$

where either $x_{1}(k)$ or $x_{2}(k)$ is cyclo-stationary signal and $\alpha_{l}=2 \pi l / p$, see Sun and Sano (2009). The cyclostationary spectrum is further defined as

$S_{x_{1}, x_{2}}\left(\alpha_{l}, \omega\right)=\sum_{\tau=-\infty}^{\infty} C_{x_{1}, x_{2}}\left(\alpha_{l}, \tau\right) e^{-i \omega \tau}$.

The over-sampling technique can also be applied in openloop identification, see Fig. 2.

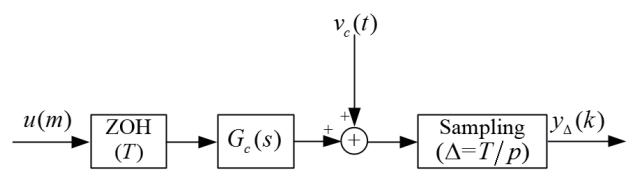

Fig. 2. Over-sampling technique in open-loop identification 


\subsection{Plant models and model conversion}

Denote the discretized plant model with respect to sampling time $T$ as

$y(m)=G\left(z^{-1}\right) u(m)+v(m)$.

where $u(m)$ and $y(m)$ are the plant input and output at sampling instant $m T$ respectively. $v(m)$ is considered as the sample of a continuous stationary stochastic process $v_{c}(t)$ at $m T$. The transfer function $G\left(z^{-1}\right)$ will be indicated as $T$-model in the sequel.

Denote the discretized plant model with respect to sampling time $\Delta$ as

$y_{\Delta}(k)=G_{\Delta}\left(q^{-1}\right) u_{\Delta}(k)+v_{\Delta}(k)$

where $u_{\Delta}(k)$ and $y_{\Delta}(k)$ are the input and output at instant $k \Delta$ respectively. Here $q^{-1}$ is a backward shift operator corresponding to the interval $\Delta \cdot v_{\Delta}(k)$ is the sample of $v_{c}(t)$ at instants $k \Delta$. The transfer function $G_{\Delta}\left(q^{-1}\right)$ will be indicated as $\Delta$-model in the sequel.

When $G_{\Delta}\left(q^{-1}\right)$ is identified, $G\left(z^{-1}\right)$ is uniquely determined by

$G\left(z^{-1}\right)=z^{-\frac{\tau_{\Delta}-1}{p}-1} c_{\Delta} \cdot\left(I-A_{\Delta}^{p} z^{-1}\right)^{-1} \sum_{j=0}^{p-1} A_{\Delta}^{j} b_{\Delta}$

where $A_{\Delta}, b_{\Delta}$ and $c_{\Delta}$ are the matrices and vectors in the state-space realization of $G_{\Delta}\left(q^{-1}\right), \tau_{\Delta}$ is the delay time of $G_{\Delta}\left(q^{-1}\right)$, see Sun and Sano (2009).

\section{The asymptotic variance expression}

Asymptotic variance expression for $\Delta$-model estimates is studied in this section. The model structure for the $\Delta$-sampled data is

$$
\begin{aligned}
& y_{\Delta}(k)=G_{\Delta}\left(q^{-1}, \theta\right) u_{\Delta}(k)+v_{\Delta}(k) \\
& v_{\Delta}(k)=H_{\Delta}\left(q^{-1}, \theta\right) e_{\Delta}(k)
\end{aligned}
$$

where $e_{\Delta}(k)$ is an i.i.d white noise. In the sequel of this section, the subscript $\Delta$ of the transfer functions is left out for simplicity, and without ambiguity all discretetime models discussed in this section are $\Delta$-models.

The prediction error for the model structure (7) is

$$
\varepsilon_{\Delta}(k, \theta)=H\left(q^{-1}, \theta\right)^{-1}\left[y_{\Delta}(k)-G\left(q^{-1}, \theta\right) u_{\Delta}(k)\right],
$$

for convenience, introduce

$$
T\left(q^{-1}, \theta\right)=\left(\begin{array}{c}
G\left(q^{-1}, \theta\right) \\
H\left(q^{-1}, \theta\right)
\end{array}\right)
$$

In this work, variance expression asymptotic in the model order is desired. However, when the model order tends to infinity, the identification test will lose informativity for parameters, but retain informativity for the system under weak conditions on the experiment design. To deal with this problem, a regularization procedure was introduced to determine the estimate $\hat{\theta}_{N}(n, \delta)$, same as in Ljung $(1985)^{2}$. Let

$\theta^{*}(n)=\underset{\theta \in D_{n}}{\arg \min } \lim _{N \rightarrow \infty} \frac{1}{N} \sum_{k=0}^{N-1} E \varepsilon_{\Delta}^{2}(k, \theta)$.

If the minimum is not unique, let $\theta^{*}(n)$ denote any, arbitrarily chosen, minimizing element. Here the argument $n$ is added to emphasize that the minimization is carried out over $n t h$ order models.

Now, define the estimate $\hat{\theta}_{N}(n, \delta)$ by

$\hat{\theta}_{N}(n, \delta)=\underset{\theta \in D_{n}}{\arg \min } V_{N}(\theta, \delta, n)$

where

$V_{N}(\theta, \delta, n)=\frac{1}{2}\left[\frac{1}{N} \sum_{k=0}^{N-1} \varepsilon_{\Delta}^{2}(k, \theta)+\delta\left|\theta-\theta^{*}(n)\right|^{2}\right]$

Here $\delta$ is a regularization parameter, helping to select a unique minimizing element in (12) in case $\delta=0$ leads to nonunique minima.

Again for convenience, introduce

$$
\begin{aligned}
& T_{n}^{*}\left(e^{-i \omega}\right)=T\left(e^{-i \omega}, \theta^{*}(n)\right) \\
& \hat{T}_{N}\left(e^{-i \omega}, n, \delta\right)=T\left(e^{-i \omega}, \hat{\theta}_{N}(n, \delta)\right) .
\end{aligned}
$$

\subsection{Assumptions}

Before deriving the asymptotic variance expression, several assumptions are made.The following assumption is made about the model structure.

Assumption 1 (Model structure) Assume that the predictor filters $H\left(q^{-1}, \theta\right)^{-1} G\left(q^{-1}, \theta\right)$ and $H\left(q^{-1}, \theta\right)^{-1}$ in (8) along with their first-, second-, and third-order derivatives with respect to $\theta$ are uniformly stable filters in $\theta$, for each given $n$.

Assume that the model structure (7) satisfies the shift property in Ljung (1985) and the model structure is identifiable.

${ }^{2}$ In Ljung (1985), the term informativity was called identifiability. 
The following assumption is made about the true system.

Assumption 2 (True system) Assume that the true $\Delta$-sampled system can be described by

$$
y_{\Delta}(k)=G_{0}\left(q^{-1}\right) u_{\Delta}(k)+H_{0}\left(q^{-1}\right) e_{\Delta}(k)
$$

where $e_{\Delta}(k)$ is a Gaussian white noise with variance $\lambda_{\Delta}$ and bounded fourth moments. Moreover, $G_{0}$ and $H_{0}$ are stable filters.

The following assumption imply the asymptotic identifiability.

Assumption 3 (Asymptotic identifiability) Assume that

$\lim _{n \rightarrow \infty} n^{2} E\left[\varepsilon_{\Delta}\left(k, \theta^{*}(n)\right)-e_{\Delta}(k)\right]^{2}=0$

which implies that $T_{n}^{*}\left(e^{-i \omega}\right)$ tends to $T_{0}\left(e^{-i \omega}\right)$ as $n$ tends to infinity.

The following assumption is made about the signals.

Assumption 4 (Signals) Assume that

$C_{u_{\Delta}, u_{\Delta}}(0, \tau)=\lim _{N \rightarrow \infty} \frac{1}{N} \sum_{k=0}^{N-1} E u_{\Delta}(k+\tau) u_{\Delta}(k)$

$C_{u_{\Delta}, e_{\Delta}}(0, \tau)=\lim _{N \rightarrow \infty} \frac{1}{N} \sum_{k=0}^{N-1} E u_{\Delta}(k+\tau) e_{\Delta}(k)$

exist and that

$C_{u_{\Delta}, e_{\Delta}}(0, \tau)=0$ for $\tau<0$

Let the spectra $S_{u_{\Delta}, u_{\Delta}}(0, \omega)$ and $S_{u_{\Delta}, e_{\Delta}}(0, \omega)$ be defined as in (3). Finally assume that

$\lim _{N \rightarrow \infty} \frac{1}{\sqrt{N}} \sum_{k=0}^{N-1} E\left[\frac{d}{d \theta} \varepsilon_{\Delta}^{2}\left(k, \theta^{*}(n)\right)\right]=0$

( for all $n$ larger than some fixed $n_{0}$ )

which implies that the limits in (16), (17) are reached sufficiently fast.

\subsection{Asymptotic variance expression}

Now the asymptotic variance expression in oversampling based identification can be derived.

Theorem 1 Let the Assumptions 1, 2, 3, 4 be in force, consider the estimate $\hat{T}_{N}\left(e^{-i \omega}, n, \delta\right)$, then

$$
\begin{gathered}
\sqrt{N}\left[\hat{T}_{N}\left(e^{-i \omega}, n, \delta\right)-T_{n}^{*}\left(e^{-i \omega}\right)\right] \in A s N(0, P(\omega, n, \delta)) \\
{[\text { as } N \rightarrow \infty \text { for fixed } n, \delta]}
\end{gathered}
$$

where

$$
\begin{aligned}
& \lim _{\delta \rightarrow 0} \lim _{n \rightarrow \infty} \frac{1}{n} P(\omega, n, \delta)= \\
& \Phi_{v_{\Delta}}(\omega)\left(\begin{array}{cc}
S_{u_{\Delta}, u_{\Delta}}(0, \omega) & S_{u_{\Delta}, e_{\Delta}}(0, \omega) \\
S_{u_{\Delta}, e_{\Delta}}(0,-\omega) & \lambda_{\Delta}
\end{array}\right)^{-1}
\end{aligned}
$$

PROOF. This result follows from Theorem 3.1 in Ljung (1985) according to which, under the assumptions, for the $\Delta$-model estimates there is

$$
\begin{gathered}
\sqrt{N}\left[\hat{T}_{N}\left(e^{-i \omega}, n, \delta\right)-T_{n}^{*}\left(e^{-i \omega}\right)\right] \in A s N(0, P(\omega, n, \delta)) \\
{[a s N \rightarrow \infty \text { for fixed } n, \delta]}
\end{gathered}
$$

and

$$
\begin{aligned}
& \lim _{\delta \rightarrow 0} \lim _{n \rightarrow \infty} \frac{1}{n} P(\omega, n, \delta)= \\
& \Phi_{v_{\Delta}}(\omega)\left(\begin{array}{cc}
\Phi_{u_{\Delta}}(\omega) & \Phi_{u_{\Delta}, e_{\Delta}}(\omega) \\
\Phi_{u_{\Delta}, e_{\Delta}}(-\omega) & \lambda_{\Delta}
\end{array}\right)-1
\end{aligned}
$$

where according to the definition of the spectrum in Ljung (1985)

$\Phi_{u_{\Delta}}(\omega)=\sum_{\tau=-\infty}^{\infty} r_{u_{\Delta}}(\tau) e^{-i \omega \tau}$

and

$r_{u_{\Delta}}(\tau)=\lim _{N \rightarrow \infty} \frac{1}{N} \sum_{t=1}^{N} E\left\{u_{\Delta}(k+\tau) u_{\Delta}(k)\right\}$.

Here in over-sampling based identification, according to the definition in (2) and under Assumption 4, the input and output signals are quasi-sationary, then there is

$\Phi_{u_{\Delta}}(\omega)=S_{u_{\Delta}, u_{\Delta}}(0, \omega)$

and similarly

$$
\begin{aligned}
& \Phi_{u_{\Delta}, e_{\Delta}}(\omega)=S_{u_{\Delta}, e_{\Delta}}(0, \omega) \\
& \Phi_{u_{\Delta}, e_{\Delta}}(-\omega)=S_{u_{\Delta}, e_{\Delta}}(0,-\omega) .
\end{aligned}
$$

Then (21) is derived.

\section{Analysis of the over-sampling scheme}

First consider the conventional closed-loop identification where the $T$-model $G\left(z^{-1}\right)$ is directly identified. According to Ljung (1985), the asymptotic variance for the $T$ model estimates in this case is

$$
\operatorname{AsVar} \hat{G}\left(e^{-i \omega}, n\right) \sim \frac{n}{N} \frac{\lambda \Phi_{v}(\omega)}{\lambda \Phi_{u}(\omega)-\Phi_{u e}(\omega) \Phi_{u e}(-\omega)},
$$


it can be further calculated in closed-loop identification as

$$
\operatorname{AsVar} \hat{G}\left(e^{-i \omega}, n\right) \sim \frac{n}{N} \frac{\Phi_{v}(\omega)}{\left|\frac{K\left(e^{-i \omega}\right)}{1+K\left(e^{-i \omega}\right) G\left(e^{-i \omega}\right)}\right|^{2} \Phi_{r}(\omega)}
$$

The expression (29) indicates that when external excitation is not applied, i.e. $\Phi_{r}(\omega)=0$, the asymptotic variance becomes infinity which means the plant model cannot be identified accurately, in other words, the identification experiment in this case is not informative.

Remark 1 Note that in the conventional case the excitation can not rely exclusively on the noise, unless the controller is of sufficient complexity (see Bazanella et.al (2010)), which is not one of the assumptions in our paper. In our case, we assume a linear finite order controller but we allow the model order to tend to infinity, which implies that the model is always more complex than the controller.

Then consider the over-sampling based closed-loop identification depicted in Fig. 1. In this case, the data length becomes $p N$ and it is reasonable to let the high order be $p n$. Then according to Theorem 1 , the asymptotic variance for $\Delta$-model estimates $G_{\Delta}\left(q^{-1}\right)$ is

$$
\begin{aligned}
& \operatorname{AsVar} \hat{G}_{\Delta}\left(e^{-i \omega}, n\right) \\
& \sim \frac{n}{N} \frac{\lambda_{\Delta} \Phi_{v_{\Delta}}(\omega)}{\lambda_{\Delta} S_{u_{\Delta}, u_{\Delta}}(0, \omega)-S_{u_{\Delta}, e_{\Delta}}(0, \omega) S_{e_{\Delta}, u_{\Delta}}(0, \omega)}
\end{aligned}
$$

And according to Theorem 1 in Sun and Sano (2009), in closed-loop identification, the cyclo-stationary spectra in (30) can be written as

$$
\begin{gathered}
S_{u_{\Delta}, u_{\Delta}}(0, \omega)=\frac{1}{p} \sum_{j=0}^{p-1} e^{-i j \omega}\left|\frac{K\left(e^{-i p \omega}\right)}{1+K\left(e^{-i p \omega}\right) G\left(e^{-i p \omega}\right)}\right|^{2} \\
\left(\Phi_{r}(p \omega)+\Phi_{v}(p \omega)\right) \sum_{j=0}^{p-1} e^{i j \omega} \\
S_{u_{\Delta}, e_{\Delta}}(0, \omega)=\frac{1}{p} \sum_{j=0}^{p-1} e^{-i j \omega} \lambda_{\Delta} \frac{-K\left(e^{-i p \omega}\right) H_{\Delta}\left(e^{i \omega}\right)}{1+K\left(e^{-i p \omega}\right) G\left(e^{-i p \omega}\right)} .
\end{gathered}
$$

Assume that the sampling period $T$ is chosen small enough to make sure that the sampling frequency is larger than the cutoff frequency of the true process $G_{0}\left(z^{-1}\right)$, then the asymptotic variance of the $T$-model $G\left(z^{-1}\right)$ estimates in over-sampling based identification can be expressed as

$\operatorname{As} \operatorname{Var} \hat{G}_{o s}\left(e^{-i \omega}, n\right) \approx \operatorname{As} \operatorname{Var} \hat{G}_{\Delta}\left(e^{-i \frac{\omega}{p}}, n\right)$

in $\omega \in[-\pi, \pi]$, with a small conversion error ignored. Note that here $\omega$ is the normalized frequency in $T$-model.
Further calculate AsVar $\hat{G}_{o s}\left(e^{-i \omega}, n\right)$ using and (32), we get

$$
\begin{aligned}
& \operatorname{AsVar} \hat{G}_{O S}\left(e^{-i \omega}, n\right) \sim \\
& \frac{n}{N} \frac{\Phi_{v_{\Delta}}\left(\frac{\omega}{p}\right)}{F\left(\frac{\omega}{p}, p\right)\left|\frac{K\left(e^{-i \omega}\right)}{1+K\left(e^{-i \omega}\right) G\left(e^{-i \omega}\right)}\right|^{2}\left(\Phi_{r}(\omega)+\left(\Phi_{v}(\omega)-\frac{1}{p} \Phi_{v_{\Delta}}\left(\frac{\omega}{p}\right)\right)\right.},
\end{aligned}
$$

where for convenience we introduce a function $F(\omega, p)$

$F(\omega, p)=\frac{1}{p} \sum_{j=0}^{p-1} e^{-i j \omega} \cdot \sum_{j=0}^{p-1} e^{i j \omega}$

and it has the property that

$F(\omega, p) \approx p$, in $\omega \in[-\pi / p, \pi / p]$

According to the over-sampling operation in Section 2, $v(m)$ is the down-sampled signal of $v_{\Delta}(k)$ by a factor of $p$, see O'Shea et al. (2011). Meanwhile, its auto-correlation function is also down-sampled by a factor of $p$, which means

$$
R_{v}(\tau)=E\left\{v_{\Delta}((m+\tau) p) v_{\Delta}(m p)\right\}=R_{v_{\Delta}}(\tau p)
$$

then according to O'Shea et al. (2011)

$\Phi_{v}(\omega)=\frac{1}{p} \sum_{k=0}^{p-1} \Phi_{v_{\Delta}}\left(\frac{\omega-2 \pi k}{p}\right)$

and when $p>1$

$\Phi_{v}(\omega)-\frac{1}{p} \Phi_{v_{\Delta}}\left(\frac{\omega}{p}\right)=\frac{1}{p} \sum_{k=1}^{p-1} \Phi_{v_{\Delta}}\left(\frac{\omega}{p}-\frac{2 \pi k}{p}\right)$.

With (36) and (39), the asymptotic variance (34) can be approximated as

$$
\begin{aligned}
& \operatorname{AsVar} \hat{G}_{O S}\left(e^{-i \omega}, n\right) \sim \\
& \frac{n}{N} \frac{\Phi_{v}(\omega)-\frac{1}{p} \sum_{k=1}^{p-1} \Phi_{v_{\Delta}}\left(\frac{\omega}{p}-\frac{2 \pi k}{p}\right)}{\left|\frac{K\left(e^{-i \omega}\right)}{1+K\left(e^{-i \omega}\right) G\left(e^{-i \omega}\right)}\right|^{2}\left(\Phi_{r}(\omega)+\frac{1}{p} \sum_{k=1}^{p-1} \Phi_{v_{\Delta}}\left(\frac{\omega}{p}-\frac{2 \pi k}{p}\right)\right)},
\end{aligned}
$$

the part $\frac{1}{p} \sum_{k=1}^{p-1} \Phi_{v_{\Delta}}\left(\frac{\omega}{p}-\frac{2 \pi k}{p}\right)$ is called the high frequency noise aliasing because it is the aliasing from the high frequency output noise beyond the bandwidth of the plant.

When no external excitation is applied, i.e. $\Phi_{r}(\omega)=0$, the expression (40) indicates that when the output contains high frequency noise, i.e., $\frac{1}{p} \sum_{k=1}^{p-1} \Phi_{v_{\Delta}}\left(\frac{\omega}{p}-\frac{2 \pi k}{p}\right) \neq 0$, 
the over-sampling based closed-loop identification can attain informative data and the plant model can be accurately identified. Therefore, using the asymptotic theory, one can give the exact informativity condition of the over-sampling based closed-loop identification without external excitation, that is when the output contains high frequency noise beyond the frequency band of the plant. An intuitive interpretation of this is that in the over-sampled system, the $T$-holding operation turns the high frequency noise into excitation in the bandwidth of the plant.

Remark 2 In Ljung (1999), the equation (13.50) states that for linear models, a closed loop experiment is not informative if there exists a linear, time-invariant, and noise-free relationship between the input and output. This can also be a interpretation for the informativity condition of the over-sampling based close-loop identification without external excitation. For the experiment in Fig. 1, when there is no external excitation and over-sampling operation is not applied, due to the feedback, we clearly have an linear relationship between the input and output, so the experiment is not informative. But when oversampling operation is applied, we cannot find an exact relationship between the input and output, since the zeroorder-held input cannot match the rapid variations in the over-sampled output. Unless the over-sampled output can be linearly reconstructed from the T-sampled output, which means the output contains no high frequency term outside the bandwidth of the plant.

Now assume that there is excitation at the set-point $r(t)$, the question is what benefits the over-sampling scheme can bring for the $T$-model?

Comparing (40) with (29), one can see two improvements using the over-sampling: (a) the term $\frac{1}{p} \sum_{k=1}^{p-1} \Phi_{v_{\Delta}}\left(\frac{\omega}{p}-\frac{2 \pi k}{p}\right)$ in the denominator of (40) acts as additional excitation, which assures the informativity in over-sampling scheme without excitation, but when there is excitation, it increases the signal-to-noise-ratio which will reduce the model variance; (b) the term $-\frac{1}{p} \sum_{k=1}^{p-1} \Phi_{v_{\Delta}}\left(\frac{\omega}{p}-\frac{2 \pi k}{p}\right)$ in the numerator of (40) means that the high frequency noise is removed in over-sampling scheme, which implies antialias and increases the signal-to-noise-ratio again.

So it has been shown that when the output contains high frequency noise, for the same test condition, using the over-sampling scheme can reduce the model variance error. Put it in another way, for the same model quality requirement, one can reduce the test signal amplitudes and/or test time using the over-sampling technique. This implies reduction of identification cost.

\section{Numerical illustrations}

\subsection{Simulation 1 - Illustration of the asymptotic vari- ance expression}

This example is to verify the asymptotic variance expression. Consider a SISO closed-loop system depicted in Fig. 1. The true process and the controller is

$G_{c}(s)=\frac{1}{2 s+1}, K\left(z^{-1}\right)=1$,

the noise system is

$v_{c}(t)=\frac{0.5 s+0.6}{s+0.6} e_{c}(t)$

here $e_{c}(t)$ is a Gaussian white noise with $N(0,1)$.

The sampling time is $T=0.4 s$, to use the over-sampling scheme, let $p=2$, thus the over-sampling period $\Delta=$ $T / p=0.2 s$. The experiment time in every simulation is $10000 T$.

According to Theorem 1, there is

$\sqrt{N}\left[\hat{G}_{N}\left(e^{-i \omega}, n\right)-G_{0}\left(e^{-i \omega}\right)\right] \in A s N(0, P(\omega, n))$.

Note that here $P(\omega, n)$ is the variance of the process model estimates only, and it is the variance for finite order. According to Theorem 1, as the order $n$ tends to infinity, this variance divided by $n$ will tend to a limit which is denoted as

$\lim _{n \rightarrow \infty} \frac{1}{n} P(\omega, n)=P_{L}(\omega)$

$P_{L}(\omega)$ can be calculated ahead of the simulation.

The ARMAX model is used to identify the transfer function $\hat{G}_{N}\left(q^{-1}, n\right)$ for $n=2,4,8$ and the actual variance $P(\omega, n)$ is obtained through 100 runs of Monte-Carlo simulations. Both situations with and without reference signal excitation have been considered here to illustrate the asymptotic variance expression.

Fig. 3 shows the plots of the simulation results $\log _{10} \frac{1}{n} P(\omega, n)$ for $n=2,4,8$ and the limit $\log _{10} P_{L}(\omega)$ when the reference signal $r(m)=0$. Fig. 4 shows the plots of the simulation results when the reference signal $r(m)$ is a Gaussian white noise with $N(0,1)$. The figures illustrate the convergence to the asymptotic variance as $n$ increases, and even for small $n$, the asymptotic variance expression gives a reasonable feel for how the exact variance changes with frequency. 


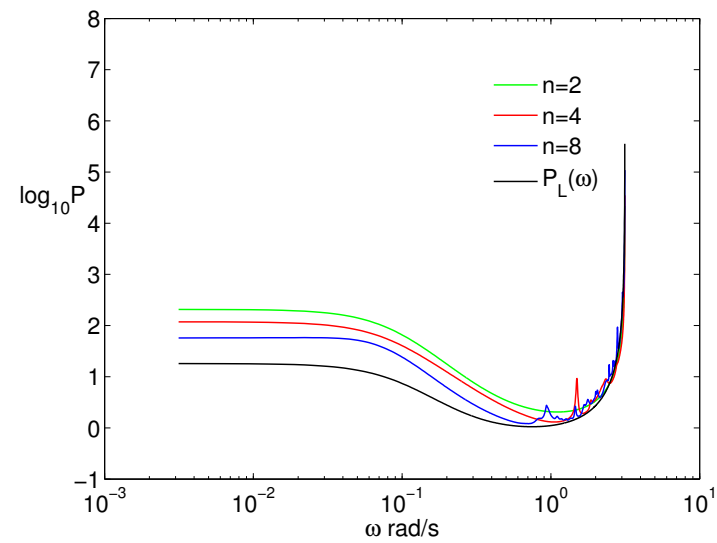

Fig. 3. Variance convergence in over-sampling case when $r(m)=0$

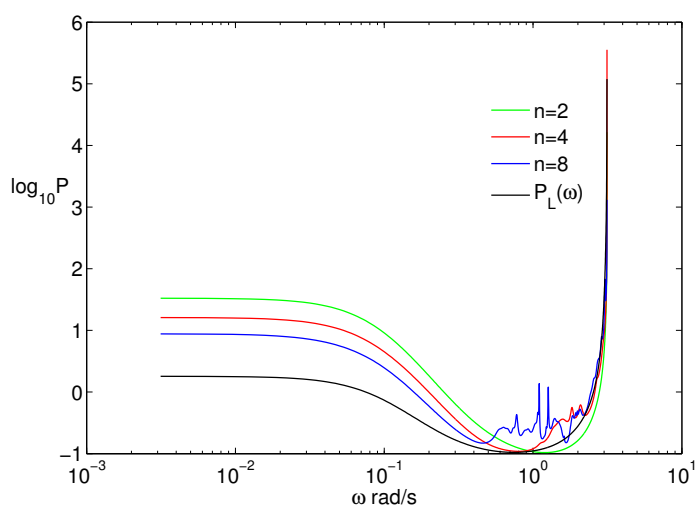

Fig. 4. Variance convergence in over-sampling case when $r(m)$ is a Gaussian white noise with $N(0,1)$

\subsection{Simulation 2 - Closed-loop identification without external excitation}

In this simulation, the same true process and controller (41) in Simulation 1 are used but with two different noise systems.

Case 1: The noise system is (42) which contains a large amount of high frequency noise, i.e. $\sum_{k=1}^{p-1} \Phi_{v_{\Delta}}\left(\frac{\omega}{p}-\frac{2 \pi k}{p}\right) \neq$ 0 .

Case 2: The noise system is $v_{c}(t)=\frac{0.6}{s+0.6} e_{c}(t)$ which contains almost no high frequency noise, i.e. $\frac{1}{p} \sum_{k=1}^{p-1} \Phi_{v_{\Delta}}\left(\frac{\omega}{p}-\frac{2 \pi k}{p}\right) \approx 0$.

In both cases no external excitation is applied, i.e. $r(m)=0$. To use the over-sampling scheme, the oversampling rate is $p=2$ and Box-Jenkins model is used to estimate the parameters. Table 1 contains the results of parameter estimation obtained through 100 runs of
Monte-Carlo simulations in both cases. The test time in every simulation is $1000 T$.

Table 1

Closed-loop identification without external excitation

\begin{tabular}{|c|c|c|}
\hline True parameters & Case 1 & Case 2 \\
\hline$a_{1}:-0.8187$ & $-0.8186 \pm 0.0233$ & $-0.6204 \pm 0.3144$ \\
\hline$b_{1}: 0.1813$ & $0.1809 \pm 0.0153$ & $0.1522 \pm 0.0717$ \\
\hline
\end{tabular}

One can see that, when the output contains high frequency noise, accurate model estimates can be obtained using the over-sampling scheme; when the output does not contain high frequency noise, the parameter estimates are very biased with large variances. These results confirm the informativity condition of closed-loop tests without external excitation in Section 4.

\subsection{Simulation 3 - Closed-loop identification with ex- ternal excitation}

Consider a closed-loop system with a second-order true process

$G_{c}(s)=\frac{0.2540 s+1.8198}{s^{2}+0.3567 s+0.2426}$

and a first-order controller

$K\left(z^{-1}\right)=\frac{1.5-0.7 z^{-1}}{1+0.5 z^{-1}}$

the zero-order holding period is $T=1 \mathrm{~s}$.

In this simulation, external excitation is applied, $r(m)$ is a Gaussian white noise. The output disturbance is

$v_{c}(t)=\sigma\left(v_{1}(t)+v_{2}(t)\right)$

where

$v_{1}(t)=\frac{5}{9 s^{2}+20 s+1} e_{1}(t), v_{2}(t)=\alpha e_{2}(t)$

$e_{1}(t)$ and $e_{2}(t)$ are independent Gaussian white noises with unit variance and zero mean, they are also independent with $r(m)$, the sampling time of them is chosen as $T / p$ to simulate continuous-time signal. $v_{1}(t)$ is a lowpass filtered color noise which contains almost no high frequency noise, the parameter $\alpha$ is to adjust the rate of white noise term in the whole output noise. The parameter $\sigma$ in (47) is used to adjust the noise-to-signal ratio. In the simulations the noise-to-signal ratio is between $10 \%$ to $20 \%$ which is normal in real applications.

Here by adjusting $\alpha$, two cases are considered:

Case 1: $\alpha=0$, the output noise contains no white noise, thus the high frequency noise is almost zero. 
Case 2: $\alpha \neq 0$, the output noise contains $50 \%$ white noise, thus the high frequency noise is not zero.

Table 2 contains the 2-norm of the standard deviations (STD) of the parameter estimates obtained in 100 runs of Monte-Carlo simulations in both cases. In every simulation, the experiment time is $1000 T$. During the simulation, it is found that the Box-Jenkins method has convergence problems when using the high frequency sampled data; it works well when using the original low frequency data. So in order to make a fair comparison, in conventional identification, the asymptotic method (ASYM, see Zhu (1998)) is used for estimation; and in over-sampling scheme an over-sampling based asymptotic method with $p=10$ is applied, see Fang and Zhu (2015).

In Table 2, "Conventional, with no noise aliasing" stands for an ideal situation where the output noise in the conventional identification tests does not contain aliasing from the high frequency band, which is equivalent to anti-aliasing filtering. According to (38), when the noise term of $v_{\Delta}(k)$ outside the frequency band $[0, \pi / p]$ is filtered out, there is $\Phi_{v}(\omega)=\frac{1}{p} \Phi_{v_{\Delta}}\left(\frac{\omega}{p}\right)$. Thus we realize this situation by letting the noise system be $v_{c}(t)=$ $\sigma\left(v_{1}(t)+\frac{1}{p} v_{2}(t)\right)$ (with sampling time $T / p$ ), while in the conventional identification (indicated as "Conventional" in Table 2) and in the over-sampling scheme (indicated as "Over-sampling"), the noise system is still (47). Note that in Case 1 the situation "Conventional, with no noise aliasing" is the same as the conventional identification since the noise system does not contain high frequency term. As for anti-aliasing low-pass filter, we found that when it is used, the obtained model is biased; and further analysis shows that the anti-aliasing filtering will result in wrong model estimate, see Fang and Zhu (2016). Therefore, anti-aliasing filter is not used in the comparison.

One can see that when the output contains high frequency noise, the over-sampling scheme increases model accuracy and outperforms the conventional method with no noise aliasing. And when the output does not contain high frequency noise, the effect of the over-sampling scheme is insignificant.

Table 2

Closed-loop identification with external excitation: 2-norm of the STD of the parameter estimates

\begin{tabular}{|c|c|c|}
\hline & Case 1 & Case 2 \\
\hline Over-sampling & 0.0085 & 0.0111 \\
\hline Conventional & 0.0093 & 0.0306 \\
\hline Conventional, with no noise aliasing & 0.0093 & 0.0134 \\
\hline
\end{tabular}

\section{Conclusion and perspective}

In this work, an asymptotic variance expression for transfer function estimates in the over-sampling scheme of identification is derived based on the result of Ljung (1985). The result holds for both open and closed-loop tests. Based on the result, we have given the exact informativity condition of Sun and coworkers' over-sampling method without excitation. Moreover, when a test signal is used, the comparison of the asymptotic variances in over-sampling scheme and in conventional identification has been conducted, and it is shown that the model errors can be reduced using the over-sampling scheme. The variance error is reduced in two ways in closed-loop over-sampling scheme: (a) turning the high frequency noise into excitation and (b) achieving antialiasing filtering. Therefore, the over-sampling scheme will outperform the conventional identification using anti-aliasing filtering. The result also has implications to continuous-time identification and to frequency domain identification. And we conject that the over-sampling scheme will in general also improve the accuracy of the disturbance model. This can be explained easily if the disturbance model is estimated from the output error residual of the plant model, because more accurate estimation of the plant model will lead to more accurate estimation of the output disturbance.

Although the asymptotic result indicates that one should use as high a sampling frequency as possible in identification, it is known that a too high sampling frequency may cause numerical problems in parameter estimation. How to balance the model accuracy and numerical difficulty needs to be addressed in future work. Another future work is to apply the asymptotic method of Zhu (1998) to over-sampling based identification; early results are very promising; see Fang and Zhu (2015).

\section{Acknowledgements}

The authors thank Lianming Sun for providing his papers on over-sampling scheme and for his useful comments on our results. We also thank reviewers for their professional critics and comments which have helped us in improving the paper.

\section{References}

[1] Bazanella, A.S., Gevers ,M. and Miskovic, L. (2010). Closedloop identification of MIMO systems: a new look at identifiability and experiment design European Journal of Control, 16(3):228 - 239.

[2] Fang, M. and Zhu, Y. (2015). Reducing Identification Error Using Over-Sampling Technique. In Proc. The 17th IFAC Symposium on System Identification, Beijing, China, October 19-21.

[3] Fang, M. and Zhu, Y. (2016). On anti-aliasing filtering and over-sampling scheme in system identification. Submitted.

[4] Ljung, L. (1985). Asymptotic variance expressions for identified black-box transfer function models. IEEE Trans. Automatic Control, 30(9):834-844. 
[5] Ljung, L. (1999). System Identification: Theory for the User. Prentice Hall, Englewood Cliffs, NJ.

[6] O'Shea, P., Sadik, A. and Hussain, Z. (2011). Digital Signal Processing. Springer Berlin Heidelberg.

[7] Söderström, T. and Stoica, P. (1989). System Identification. Prentice Hall Int.

[8] Sun, L., Wen, L. and Sano, A. (1997). Over-sampling approach to closed-loop identification. In Proc. 36th Conference on Decision \& Control. San Diego, California USA, December.

[9] Sun, L., Ohmori, H. and Sano, A. (2000). Frequency domain approach to closed-loop identification based on output intersampling scheme. In Proceedings of the American Control Conference. Chicago, Illinois, June.

[10] Sun, L., Ohmori, H. and Sano, A. (2001). Output intersampling approach to closed-loop identification. IEEE Trans. Automatic Control, 46(12):1936-1941.

[11] Sun, L. and Sano, A. (2009). Over-sampling approach to direct closed-loop identification and its performance. In Proc.15th IFAC Symposium on System Identification, Saint-Malo, France.

[12] Sun, L. and Zhu, Y. (2012). New Closed-loop identification approach based on output over-sampling scheme. In Proc.17th IFAC Symposium on System Identification, Brussels, Belgium.

[13] Wang, J., Chen, T. and Huang, B. (2004). Closed-loop identification via output fast sampling. Journal of Process Control, 14:555-570.

[14] Zhu, Y. (1998). Multivariable process identification for MPC: the asymptotic method and its applications. Journal of Process Control, 8(2):101-115.

[15] Zhu, Y. (2001). Multivariable System Identification for Process Control. Pergamon. 